\title{
Author Correction: Metal-coordinated sub-10 nm membranes for water purification
}

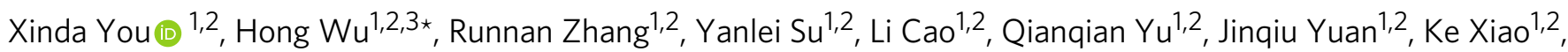
Mingrui $\mathrm{He}^{1,2} \&$ Zhongyi Jiang ${ }^{1,2 \star}$

Correction to: Nature Communications https://doi.org/10.1038/s41467-019-12100-0, published online 12 September 2019.

The original version of this Article contained errors in Fig. 4 and Supplementary Fig. 5. In Fig. 4a, some of the permeance data in each of the three solutions were inadvertently incorrectly transferred from the original data sheets to the plotting software.

The correct version of Fig. $4 \mathrm{a}$ is
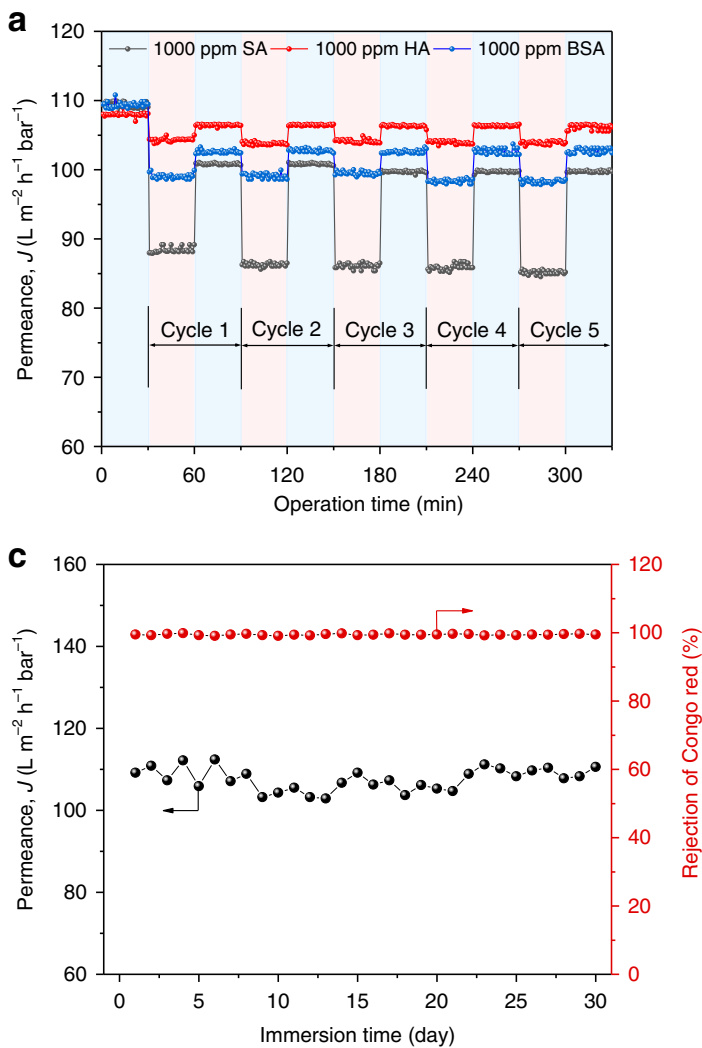

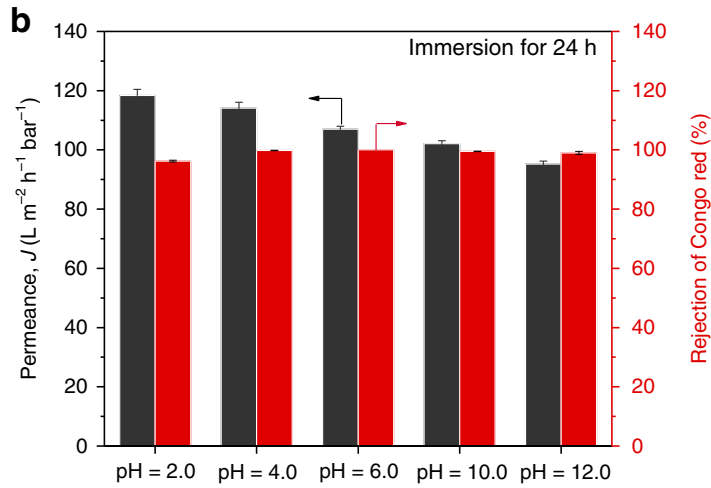

d

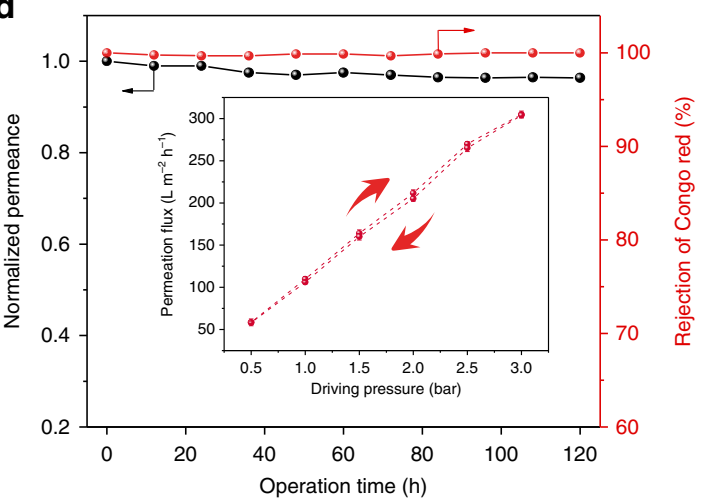

\footnotetext{
${ }^{1}$ Key Laboratory for Green Chemical Technology, School of Chemical Engineering and Technology, Tianjin University, Tianjin 300072, China. ${ }^{2}$ Collaborative Innovation Center of Chemical Science and Engineering (Tianjin), Tianjin 300072, China. ${ }^{3}$ Tianjin Key Laboratory of Membrane Science and Desalination Technology, Tianjin University, Tianjin 300072, China. *email: wuhong@tju.edu.cn; zhyjiang@tju.edu.cn
} 
The incorrect original version of Fig. $4 \mathrm{a}$ is
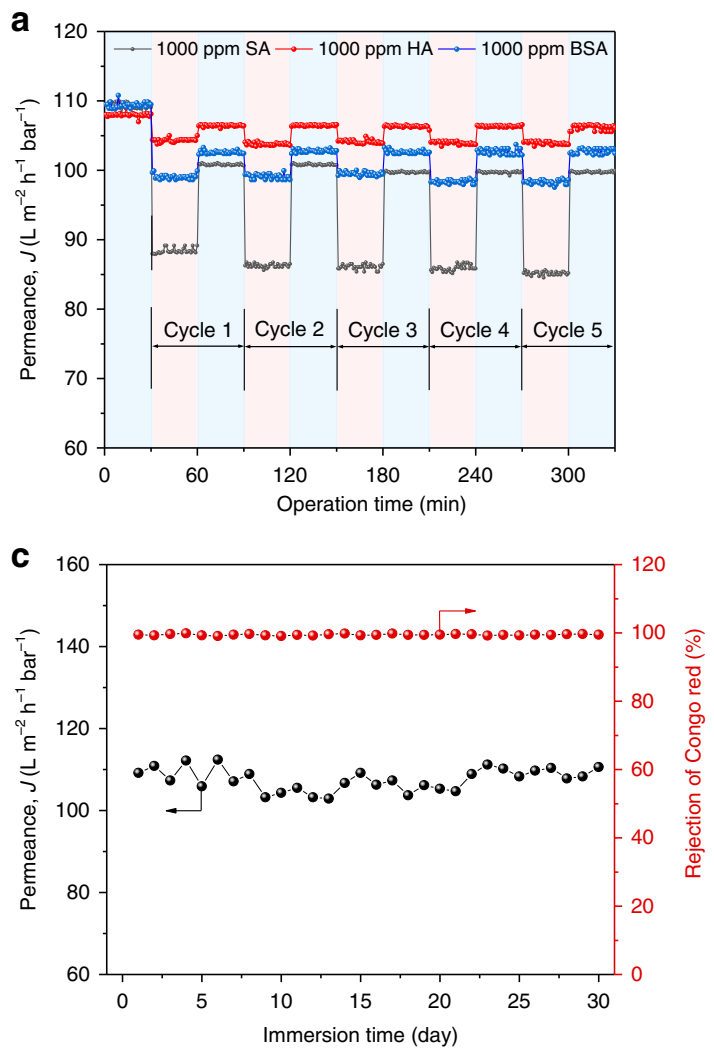

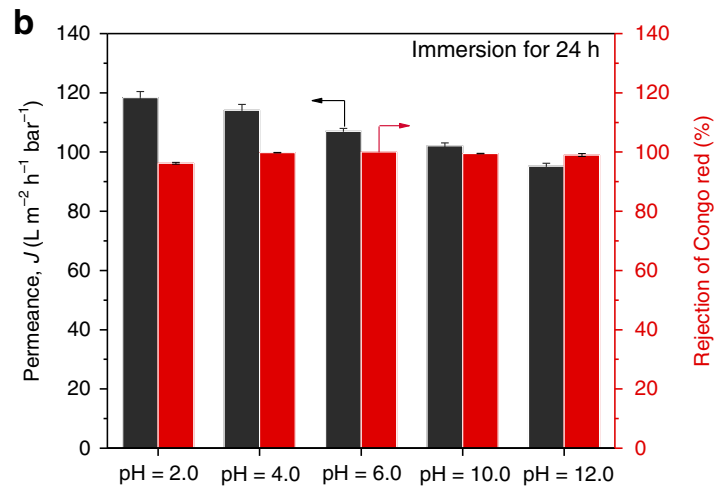

d

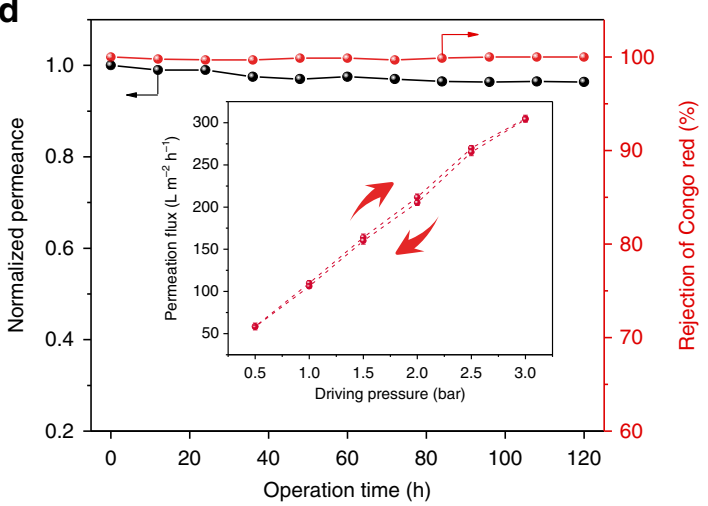

This has been corrected in both the PDF and HTML versions of the Article.

In Supplementary Fig. 5, the SEM image of MOPM- $\mathrm{Ni}^{2+}$ was inadvertently replaced by a duplicate of MOPM- $\mathrm{Zn}^{2+}$. The HTML has been updated to include a corrected version of the Supplementary Information.

Published online: 08 November 2019

cc (i) Open Access This article is licensed under a Creative Commons Attribution 4.0 International License, which permits use, sharing, adaptation, distribution and reproduction in any medium or format, as long as you give appropriate credit to the original author(s) and the source, provide a link to the Creative Commons license, and indicate if changes were made. The images or other third party material in this article are included in the article's Creative Commons license, unless indicated otherwise in a credit

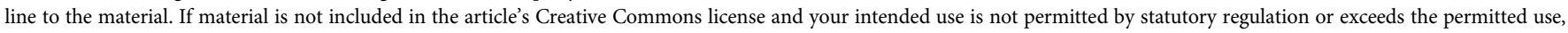
you will need to obtain permission directly from the copyright holder. To view a copy of this license, visit http://creativecommons.org/licenses/by/4.0/.

(C) The Author(s) 2019 